\title{
Local star-forming dwarf galaxies as windows on reionization-era stellar populations
}

\author{
Peter Senchyna \\ University of Arizona / Steward Observatory, USA
}

\begin{abstract}
The recent detections of high-ionization nebular line emission from species including CIV in a number of $z>6$ galaxies have highlighted substantial deficiencies in our understanding of metal poor stars. Prominent nebular CIV has never been detected in purely star-forming systems locally, and the massive star models used to model this emission in photoionization codes have not been empirically calibrated below the metallicity of the SMC ( $20 \%$ solar). As a result, we are presently entirely unprepared to correctly interpret nebular emission from metal-poor stars observed with JWST and ALMA in the reionization era. We present results from a multipronged ongoing local ultraviolet/optical observation campaign with HST/COS, Keck/ESI, and MMT designed to address this issue by locating and characterizing stellar populations capable of powering such high-ionization emission. This work has already demonstrated that strong nebular CIV can be powered by extremely metal-poor $(<10 \%$ solar $)$ massive stars, indicating that we may already have evidence of such low-metallicity populations in the reionization era. However, CIV at the equivalent widths detected at $z>6$ remains elusive locally, potentially in part due to the relative paucity of known nearby galaxies at these metallicities with massive stellar populations comparable to those in $z>6$ systems. We present a new technique to locate such nearby galaxies, and results from optical follow-up which indicate that a substantial population of highly star- forming metal-poor galaxies likely resides just below the detection limits of previous large spectroscopic surveys.
\end{abstract}

